\title{
A detailed analysis of the optical beam deflection technique for use in atomic force microscopy
}

\author{
Constant A. J. Putman, ${ }^{\text {a) }}$ Bart G. De Grooth, Niek F. Van Hulst, and Jan Greve \\ Department of Applied Physics, University of Twente, P.O. Box 217, 7500 AE Enschede, The Netherlands
}

(Received 7 October 1991; accepted for publication 30 March 1992)

\begin{abstract}
A Michelson interferometer and an optical beam deflection configuration (both shot noise and diffraction limited) are compared for application in an atomic force microscope. The comparison shows that the optical beam defiection method and the interferometer have essentially the same sensitivity. This remarkable result is explained by indicating the physical equivalence of both methods. Furthermore, various configurations using optical beam deflection are discussed. All the setups are capable of detecting the cantilever displacements with atomic resolution in a $10 \mathrm{kHz}$ bandwidth.
\end{abstract}

\section{INTRODUCTION}

The atomic force microscope (AFM) was introduced by Binnig, Quate, and Gerber ${ }^{1}$ as a method for highresolution topographic imaging of both insulators and conductors. In the AFM the sample to be imaged is brought close to a sensing tip attached to a small cantilcver. Interaction forces between sample and tip deflect the cantilever. In the original design, the cantilever displacement was detected by a tunneling tip on the back of the cantilever. ${ }^{1}$ Subsequently various optical techniques have been developed for the displacement detection. These techniques include diode laser feedback detection, ${ }^{2,3}$ interferometry, ${ }^{4-9}$ and optical beam deflection. ${ }^{10,11}$

The optical beam deflection technique introduced by Meyer and $\mathrm{Amer}^{10}$ is a very reliable and simple detection method. Because of this, the optical beam deflection method is widely used in laboratory and commercially available AFMs. ${ }^{12,13}$ The cantilever displacement is measured by detecting the deflection of a laser beam reflected from the backside of the cantilever. The deflection is detected by a position sensitive detector (split detector).

At first sight it is somewhat surprising that such a simple method yields similar results as the more complex interferometers and is sensitive enough to obtain atomic resolution. Therefore, we have performed a detailed theoretical analysis of the optical beam deflection method in various configurations. The analysis shows that the signalto-noise ratios (SNR), assuming shot noise limited performance of the detection systems, are sufficiently high to achieve atomic resolution.

A comparison shows that the SNR of the optical beam deflection method is comparable to that of interferometric techniques. This is explained by indicating the physical equivalence of both techniques.

\section{OPTICAL BEAM DEFLECTION VS INTERFEROMETRY}

For the detection of the cantilever displacements in AFM, the fundamental limits of any optical detection sys-

a) To whom correspondence should be directed. tem are set by diffraction and photon shot noise. For the detection system as such, other sources of noise, e.g., mechanical and acoustic vibrations, electronic noise, light source instabilities, and thermal noise, are of a less fundamental nature and will be discussed briefly in the discussion. In this section we will give an idealized description of the optical beam deffection and an interferometer, assuming shot noise and diffraction limited performance.

Let us first consider a Michelson-type interferometer which is widely used in AFM. The response of the interferometer as a function of the tip position in the idcal case (fringe visibility is 1 ) is shown in Fig. 1. At quadrature (point $Q$ ), for small displacements of the tip $\Delta z$, the change in the number of photons incident on the detector during a measurement (duration: $t \mathrm{~s}$ ) can be approximated by

$$
\Delta N=t N_{\text {tot }} \Delta z 2 \pi / \lambda,
$$

where $N_{\text {tot }}$ is the total amount of photons emitted by the laser per second and $\lambda$ is the wavelength of the laser light. The signal-to-noise ratio (SNR) for the measurement of $\Delta z$, in terms of photons incident on an ideal detector (quantum efficiency of 1 and no dark count), is given by

$$
\mathrm{SNR}_{\mathrm{inf}}=\left(8 \pi^{2} t N_{\mathrm{tot}}\right)^{1 / 2} \Delta z / \lambda,
$$

where it is assumed that the value of the equilibrium state (quadrature) has been established over a long period of time to minimize the uncertainty in that signal.

In order to obtain a similar analysis for optical beam deflection, we consider the next idealized situation. A collimated Gaussian laser beam with diameter $D_{0}$ is incident on the back side of a cantilever (length $l, D_{0}<l$ ) that has a reflective flat surface. The reflected beam is focused by a lens (focal length $f$ ) on a position sensitive detector (Fig. 2 ), consisting of two closely spaced detectors (split detector). The assumption is made that the width of the gap between the two detectors is small compared to the size of the laser beam at the detector. We further assume here that $D_{0}<l$ so that almost all the light is reflected by the cantilever. In the focal plane of the lens the intensity distribution is also Gaussian with a diameter ${ }^{14}$ 


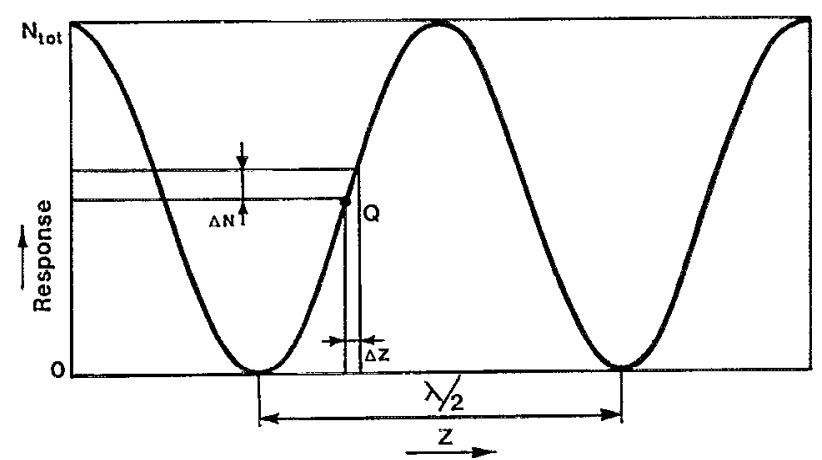

FIG. 1. The response of an interferometer expressed as the number of photons incident on the detector per second as function of the tip position, $z$. At quadrature, point $Q$, a change in cantilever position $\Delta z$, causes a change in the amount of photons incident on the detector, $\Delta N$.

$$
D_{\mathrm{sd}}=\frac{4 \lambda f}{\pi D_{0}} .
$$

A displacement of the cantilever $\Delta z$ results in a displacement of the laser spot on the split detector, $\Delta s$. We assume that the cantilever rotates around its base as a solid body and that there is no bending of the cantilever. In that case $\Delta s$ is given by

$$
\Delta s=2 f \frac{\Delta z}{l} \text {. }
$$

The factor 2 is due to the fact that the angular deflection of the reflected laser beam is twice the angular deflection of the cantilever. At first sight one might expect that only displacements larger than the laser spot can be detected (Rayleigh criterium ${ }^{15}$ ). This, however, is not true. If the split detector is positioned at the focal plane of the lens, much smaller displacements can be detected by substracting the signals of the two detectors. The detection limit is determined by the accuracy of the difference signal and in the ideal case considered here by the number of photons detected. This is illustrated in Fig. 3. This difference signal, in terms of photons incident on the detector, can be calcu-

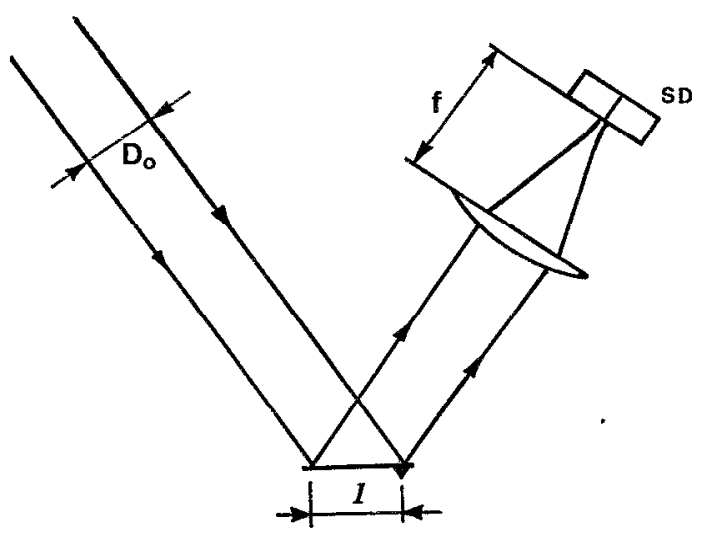

FIG. 2. A collimated laser beam (diameter $D_{0}$ ) is incident on the cantilever (length $l$ ). The reflected light is focused by a lens (focal length $f$ ) onto the split detector SD.

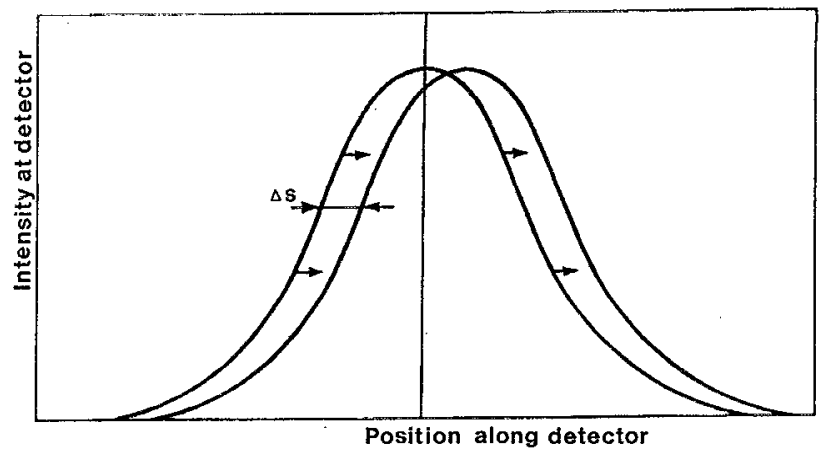

FIG. 3. In optical beam deflection a small tip displacement $\Delta z$ causes a change $\Delta s$ in the position of the intensity distribution at the split detector. This small displacement is detected by substracting the signals of the two segments of the detector.

lated using Eqs. (3) and (4) and the fact that the irradiance profile at the split detector is Gaussian

$$
\Delta N=t N_{\text {tot }} 2(2 \pi)^{1 / 2} \frac{D_{0}}{l} \frac{\Delta z}{\lambda},
$$

where the numerical factor is due to the Gaussian shape of the laser beam (see Appendix A) and diffraction. The SNR can now be calculated

$$
\mathrm{SNR}_{\mathrm{obd}}=\frac{\Delta N}{\left(t N_{\text {tot }}\right)^{1 / 2}}=\left(8 \pi t N_{\mathrm{tot}}\right)^{1 / 2} \frac{D_{0}}{l} \frac{\Delta z}{\lambda} \text {. }
$$

This equation suggests that $D_{0}$ should be made as large as possible in order to maximize the SNR. However, our analysis is only valid for $D_{0}<l$. For $D_{0}>l$ diffraction of the laser beam at the cantilever will disturb the Gaussian irradiance distribution of the reflected beam. This situation will be discussed in Sec. V.

In the limiting case $\left(D_{0}=l\right)$, when the whole cantilever is used, the ratio between $\mathrm{SNR}_{\text {inf }}$ and $\mathrm{SNR}_{\mathrm{obd}}$ is $\pi^{1 / 2}: 1$ [ratio between Eqs. (2) and (6)]. This leads to the remarkable conclusion that for the ideal cases, in terms of sensitivity, the optical beam deflection method and the interferometer are equivalent. In Ref. 16 we have made this plausible by indicating the physical equivalence of both methods (see also Sec. VI).

In the next sections various setups using optical beam deflection, less ideal than presented here but more realistic, will be discussed.

\section{A COLLIMATED LASER BEAM ON THE CANTILEVER}

Meyer and Amer ${ }^{10}$ introduced the optical beam deflection technique using a collimated beam from a $\mathrm{HeNe}$ laser which was incident on a relative large square mirror attached to the back of a tungsten cantilever. The setup in that situation is the same as in the previous section, except for the focusing lens in front of the detector which was omitted. A derivation of the SNR in this case leads to

$$
\mathrm{SNR}_{2}=\left(\frac{\lambda \eta P_{\mathrm{tot}}}{2 h c B}\right)^{1 / 2} 2 g_{1} g_{2} \frac{X}{D(X)} \frac{\Delta z}{l},
$$


where $P_{\text {tot }}$ is the total optical power incident on the detector, $\eta$ the quantum efficiency of the detector, $h$ Plancks constant, $c$ speed of light in air, $B$ the bandwidth of the detection system, $X$ is the distance between cantilever and detector, and $g_{2}$ is a geometrical factor and equal to $4(\pi /$ 2) ${ }^{1 / 2}$ for Gaussian laser beams (see Appendix A). The geometrical factor $g_{1}$ takes the gradual bending of the cantilever into account. ${ }^{17-19}$ Depending on the position of the laser spot on the cantilever, it can have a value between 0 (base) and 2 (top). The actual angular deffection is obtained when $\Delta z / l$ is multiplied by $g_{1}$.

The conclusion could be drawn, that by making $X$ as large as possible the SNR would reach an unlimited value. The diameter of the collimated laser beam $D(X)$, however, is not constant when $X$ becomes large. Given a laser beam waist (diameter $D_{0}$ ) the diameter of a Gaussian laser beam as a function of $X$ is given by ${ }^{14}$

$$
D(X)=D_{0}\left\{1+\left(\frac{4 \lambda X}{\pi D_{0}^{2}}\right)^{2}\right\}^{1 / 2}
$$

$\mathrm{SNR}_{2}$ is maximized when $X$ becomes very large and approaches a diffraction limited value for $X>\pi D_{0}^{2} / 4 \lambda$ and the ratio $X / D(X)$ is fixed by diffraction. In that case, Eq. (7) reduces to Eq. (6) if Eq. (6) is rewritten using the optical power instead of photons incident on the detector

$$
\mathrm{SNR}_{1}=\left(\frac{\lambda \eta P_{\mathrm{tot}}}{2 h c B}\right)^{1 / 2} g_{1} g_{2} \frac{\pi}{2} \frac{D_{0}}{l} \frac{\Delta z}{\lambda}
$$

Thus from the above analysis it is clear that, when using a collimated laser beam, the distance between cantilever and detector should be large: $X>\pi D_{0}^{2} / 4 \lambda$. The limiting case can also be obtained by positioning a focusing lens in front of the detector (see previous section). Since both $\Delta s$ and $D_{\mathrm{psd}}$ (diameter of the lascr spot at the detector) are proportional to $f$, the above analysis shows that the designer of an AFM has a freedom of choice with respect of the focal length of the lens used.

\section{LASER BEAM FOCUSED ON THE CANTILEVER}

A major drawback of the configurations using collimated laser beams, is the fact that large reflective surfaces are required to direct the reflected laser beam towards the detector. This can be done by attaching a mirror to the back of the cantilever. ${ }^{10,11}$ Recently, microfabricated cantilevers ${ }^{20}$ which have a reflective gold coating on the backside have become commercially available. ${ }^{12,13}$ The cantilevers have a triangular form with a base-height ratio of about 1 and the dimensions are small, typically 100 or 200 $\mu \mathrm{m}$. Since the cantilever has a triangular hole at the base, the area that can be used effectively for the reflection of the laser beam is about $50 \mu \mathrm{m}$ in diameter. Due to diffraction it is not possible to create a laser beam of that size which stays collimated over a wide range. A laser beam with a waist of $50 \mu \mathrm{m}$ and a wavelength of $780 \mathrm{~nm}$ doubles its size after only $5 \mathrm{~mm}$ [see Eq. (8)]. Thus it is clear that collimated laser beams can be used no longer in that situation. In this and the next section two alternative configurations which can be used for small cantilevers will be discussed.

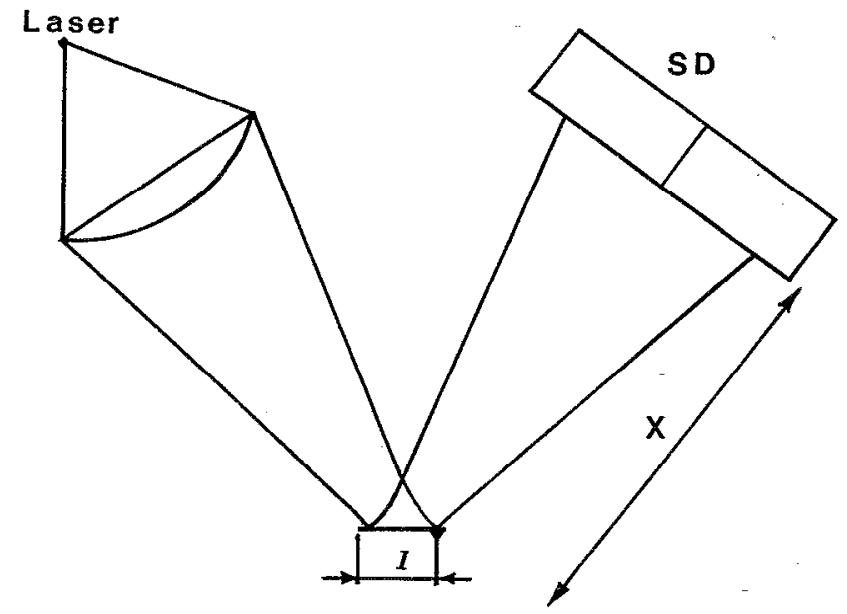

FIG. 4. A beam from a diode laser is focused at the cantilever (length $l$ ). The reflected beam is detected by the split detector SD. Distance between cantilever and detector is $X$.

We first consider the situation in which the Gaussian laser beam is focused on the cantilever as shown in Fig. 4. In that case, optical power loss is small. Assuming that the detector is positioned far from the waist region [Eq. (3) is valid], the relative displacement of the Gaussian laser spot at the detector can be written as

$$
\frac{\Delta s}{D_{\mathrm{psd}}}=g_{1} \frac{\pi}{2} \frac{D_{0}}{l} \frac{\Delta z}{\lambda}
$$

where $D_{0}$ is the spot diameter on the cantilever. Having obtained this equation and using Eq. (A3) in Appendix A, some mathematics results in

$$
\mathrm{SNR}_{3}=\left(\frac{\lambda \eta P_{\text {tot }}}{2 h c B}\right)^{1 / 2} g_{1} g_{2} \frac{\pi}{2} \frac{D_{0}}{l} \frac{\Delta z}{\lambda} .
$$

This equation is the same as $\mathrm{SNR}_{1}$ [Eq. (9)], the expression describing the setup using a collimated laser beam. When examining the two configurations more closely, it is clear that they are comparable. Only the waist region in the setup with the laser beam focused on the cantilever is much shorter and the limiting case (restricted by diffraction) is already reached when the detector is at a very close distance from the cantilever. The SNR for intermediate cases (detector position from waist region to the far field) can be calculated using Eqs. (7) and (8).

As can be seen from Eq. (11), the SNR is independent of the distance $X$ between cantilever and detector (as long as the spot size is smaller than the detector size and the detector is positioned far from the waist region). The geometrical amplification factor of $800\left(2 X / l\right.$, Drake et al. $\left.{ }^{21}\right)$ is not as important as it seems at first sight. It is true that the displacement of the spot at the detector is proportional to $X$, but that holds also for the diameter of the spot. As mentioned before, this ratio is fixed by diffraction. Positioning a focusing lens in front of the detector will not increase the sensitivity because the ratio $\Delta s / D_{\text {psd }}$ is already constrained by diffraction. In fact, the SNR will decrease. In the case when the cantilever is imaged at the detector, 


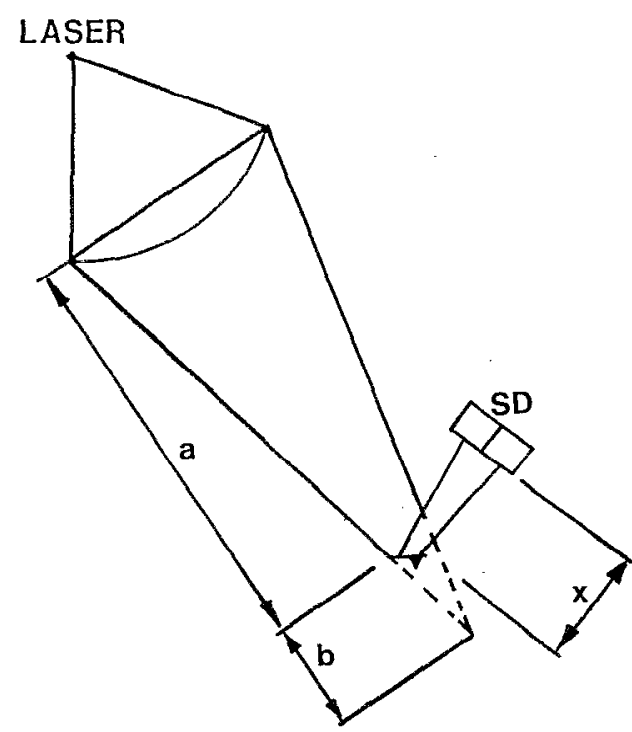

FIG. 5. The laser beam is focused at the detector. $a$ : distance lenscantilever, $b$ : distance cantilever-focus, $X$ : distance cantilever-detector. Only part of the laser beam is reflected by the small cantilever.

no signal will be detected at all. This is in contrast to the configuration with the collimated laser beam.

\section{LASER BEAM FOCUSED ON THE PSD}

As a second case the situation where the laser beam is focused at the detector will be considered. In previous sections we have found that the SNR is proportional to the beam displacement at the detector relative to the diameter of the beam at that position. Thus focusing the beam at the detector could increase the SNR. On the other hand, the dimensions of the microfabricated cantilever are such that only the central part of the Gaussian laser beam will be reflected, as is shown in Fig. 5. This means that only a fraction of the available optical power is used. Moreover, due to diffraction the size of the light spot at the detector increases. Both effects result in a decrease of the SNR. Thus, in order to obtain an expression for the SNR in this case, we have to take these effects into account.

For sake of simplicity we approximate the laser beam at the cantilever by plane waves. The reflective surface of the cantilever is approximated by a circular disk (diameter $D_{c}$ ). It is assumed that the diffraction of the reflected waves is dominated by the small dimensions of this disk. This assumption is valid if the diameter of the laser beam at the cantilever is much larger than $D_{c}$. If the laser beam is almost perpendicular to the cantilever the optical power that is reffected towards the detector can be approximated by

$$
P_{\text {det }}=2 \frac{(a+b)^{2}}{b^{2}} \frac{D_{c}^{2}}{D^{2}} P_{\text {tot }}
$$

where $D$ is the diameter of the laser beam at the lens, $a$ is the separation between lens and cantilever, and $b$ between cantilever and position of the focus (see Fig. 5). The factor 2 is due to the fact that the maximum intensity in a Gauss- ian laser beam is twice the average intensity. In Appendix $B$ it is shown that the size of the light spot on the detector is given by the diameter of the first Fresnel zone, $D_{\text {psd }}$ $=2(\lambda X)^{1 / 2}(X$ is the distance between cantilever and detector). ${ }^{15}$ The relative displacement is given by

$$
\frac{\Delta s}{D_{\mathrm{psd}}}=g_{1}\left(\frac{X}{\lambda}\right)^{1 / 2} \frac{\Delta z}{l} \text {. }
$$

Using Eqs. (12), (13), and (B3) (Appendix B) and setting $X$ equal to $b$, an expression for SNR can be derived

$$
\mathrm{SNR}_{4}=\left(\frac{\lambda \eta P_{\text {tot }}}{2 h c B}\right)^{1 / 2} g_{1} g_{3} \frac{a+b}{b} \frac{D_{c}}{D}\left(\frac{2 b}{\lambda}\right)^{1 / 2} \frac{\Delta z}{l},
$$

where $g_{3}$ is a geometrical factor and is equal to $8 / \pi$ (see Appendix B).

A variation of the previous scheme is obtained when the reflected light is collected by a lens and focused onto the detector. In that case the situation can be described by Fraunhofer diffraction theory. ${ }^{15}$ An Airy disk is formed at the detector with the diameter of the central spot

$$
D_{\text {airy }}=2.44 \frac{\lambda f}{D_{c}} \text {. }
$$

Using this equation and Eq. (12) (see also last remark in Appendix B) the SNR is

$$
\mathrm{SNR}_{5}=\left(\frac{\lambda \eta P_{\text {tot }}}{2 h c B}\right)^{1 / 2} 0.58 g_{1} g_{2} \frac{a+b}{b} \frac{D_{c}}{D} \frac{D_{c}}{l} \frac{\Delta z}{\lambda}
$$

As before, the SNR is not a function of $f$.

The practical use of the setups in this section is not apparent at first sight. But if one wishes to build an AFM in which the tip and not the sample is scanned, the laser spot at the cantilever should be large enough to minimize the fluctuations in the detector signal caused by the cantilever movement. Another solution would be to scan the laser beam simultaneously.

\section{DISCUSSION}

In the second section we have compared the SNR of optical beam deflection to the SNR of an interferometer. We came to the conclusion that the precision of cantilever displacement measurement of the two techniques are equivalent. It can be made plausible that the physical principles underlying both methods are similar and that optical beam deflection in essence is also an interferometric technique. In the following discussion we restrict ourselves to the situation described in Sec. II, where the size of the cantilever is large. Suppose we split the collimated laser beam into two collimated beams, one incident at the base of the cantilever and the other at the top of the cantilever (Fig. 6). The lens causes the two beams to interfere at the detector and an interference pattern will be formed. A tip displacement causes a change in phase difference between the two beams which results in a shift of the interference pattern at the detector. By an optimal measurement of the change in interference pattern, phase differences introduced by cantilever displacements are detected. 'The two beams can be considered as the outer rays of the collimated 


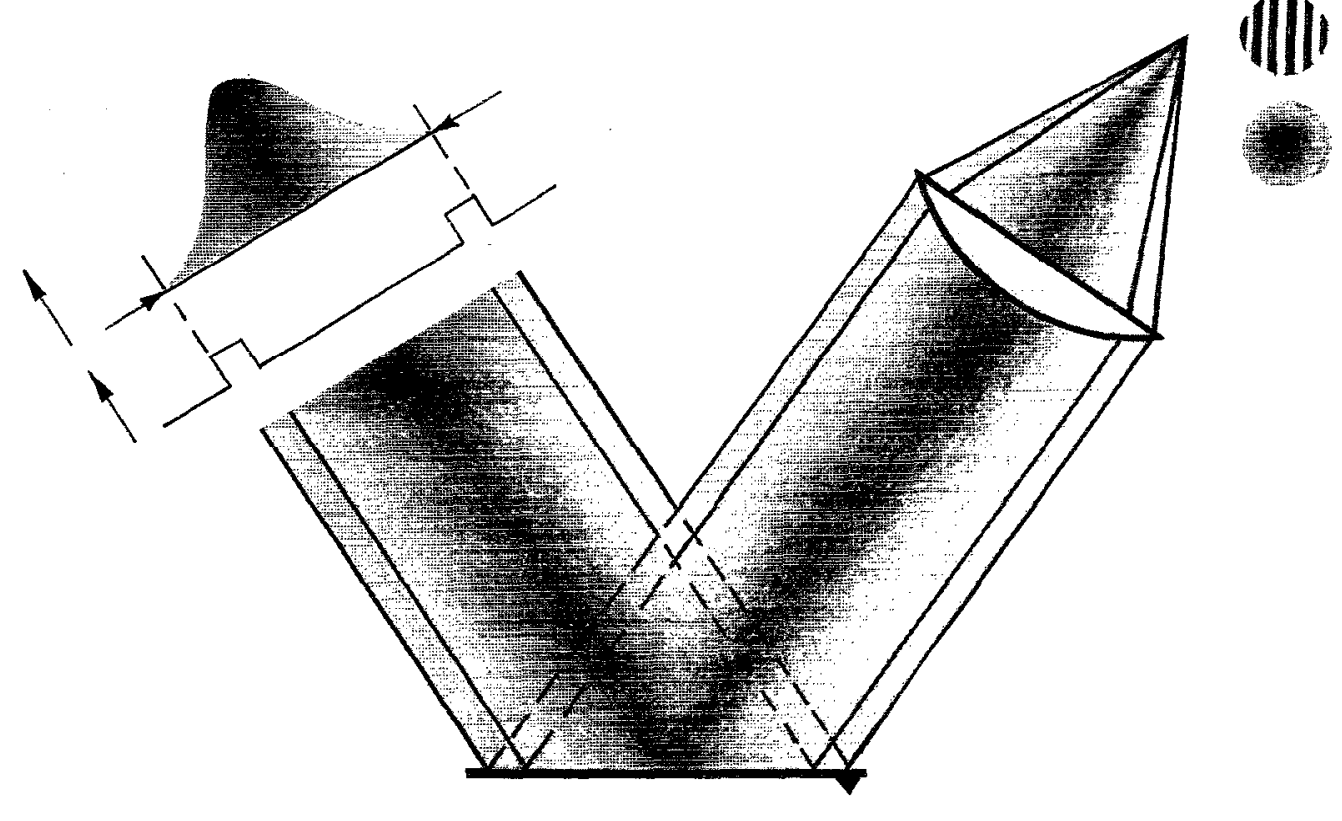

FIG. 6. Hypothetical setup of the optical beam deflection using two collimated laser beams. In the focal plane of the lens the two beams interfere and the interference pattern as shown on the upper right side will be formed. This pattern shifts if the cantilever position changes. A single Gaussian laser beam results in a Gaussian interference pattern (second pattern). Cantilever displacement causes a shift of this pattern which can be measured by a split detector.

laser beam actually used in optical beam deflection. In addition to these rays numerous other rays form the Gaussian intensity profile. In the focal plane of the lens at the detector, all these rays interfere and form an interference pattern which is again a Gaussian spot. A tip displacement results in a change of the interference pattern which is now a simple shift of the Gaussian beam waist. This shift is detected by a split detector. In this way the optical beam deflection technique can be considered as an interferometric technique.

Setting SNR $_{\text {inf }}$ [Eq. (2)] equal to 1 and rewritting the equation gives

$$
\frac{2 \pi}{\lambda} 2 \Delta z_{\min }=\frac{1}{\left(t N_{\text {tot }} / 2\right)^{1 / 2}}
$$

where $\Delta z_{\min }$ is the minimum detectable cantilever displacement. The term on the left-hand side can be substituted by $\Delta \phi$, the uncertainty in phase difference between the two interfering beams. The square-root term on the right-hand side is substituted by $\Delta n$, the uncertainty in the number of photons incident on the detector (quadrature). Equation (17) becomes

$$
\Delta \phi \Delta n=1 \text {. }
$$

This is in agreement with the uncertainty relation for the harmonic oscillator, $\Delta \phi \Delta n \geqslant 1$, where $\phi$ is the phase of the oscillator and $n$ the number of quanta. ${ }^{22}$ Moreover, we thus see that the Michelson interferometer is an optimal method for detecting phase differences between two beams.

The sensitivity of the optical beam deflection technique depends on three important factors. Firstly, the difference of the optical path length introduced by a cantilever dis- placement should be maximized by illuminating the cantilever surface $\left(D_{0} / l \cong 1\right)$ totally. This effect can be amplified by attaching a large elongated mirror to the cantilever. Secondly, the interference of the rays coming from various points of the cantilever should be optimal. When using normal imaging optics this is obtained in the Fourier plane. In the case of a focusing lens in front of the detector (Sec. II) this is in the focal plane of the lens. Using no lens (Secs. III and IV), this is in the far-field region. Thirdly, the total optical power available should be used.

We have derived the SNRs for various setups using optical beam deflection. The minimum detectable displacements $(\Delta z)_{\min }$ of the setups are compared in Table I using practical numerical values for the parameters involved. Re-

TABLE I. Comparison of the sensitivity of the optical displacement detection techniques. Setup ${ }_{1}$ : collimated laser on the cantilever plus focusing lens in front of detector. Setup ${ }_{3}$ : laser beam focused at the cantilever. Setup $_{4}$ : laser beam focused at the detector. Setups: Same as setup $p_{4}$ but with a focusing lens in front of the detector. $(\Delta z)_{\min }$ is in $\mathrm{m} / \mathrm{Hz}^{1 / 2}$. The numerical values used: $\eta=0.8, P_{\text {tot }}=1 \mathrm{~mW}, \lambda=780 \mathrm{~nm}, g_{1}=1.5$ (for all expressions) and $D_{0} / l=1 / 3$ [Setup ${ }_{1}$ Eq. (9)], $D_{0} / l=1 / 3$ [setup 3 , Eq. (11)], $a / b=10, D=4 \mathrm{~mm}, D_{c}=50 \mu \mathrm{m}, X=5 \mathrm{~mm}$ and $l=200 \mu \mathrm{m}$ [setup 4 , Eq. (14) and $\operatorname{setup}_{5}$, Eq. (16)].

\begin{tabular}{lc}
\hline \hline & $(\Delta z)_{\min }$ \\
\hline Setup $_{1,3}$ & $7.9 \times 10^{-15}$ \\
Setup $_{4}$ & $8.5 \times 10^{-14}$ \\
Setup $_{5}$ & $2.1 \times 10^{-13}$ \\
Optical beam deflection (Ref. 18) & $1.0 \times 10^{-13}$ \\
Interferometry (Ref. 5) & $1.7 \times 10^{-13}$ \\
Fiberinterferometry (Ref. 9) & $5.5 \times 10^{-14}$ \\
Laser diode feedback (Ref. 3) & $3.0 \times 10^{-12}$ \\
\hline \hline
\end{tabular}


ported measured sensitivities of various optical displacement detection systems are also included. As we have seen in Sec. IV, the sensitivity of the setups using a collimated laser beam or a focused laser beam on the cantilever is the same. This is due to the fact that the change in optical path length is the same since in both cases the same relative area of the cantilever is occupied by the laser beam. The sensitivity of the setups in which the cantilever reflects only a central part of the Gaussian laser beam ( setups $_{4,5}$ ) is a factor 10 less than setup ${ }_{1,3}$. This is due to the fact that not all available optical power is used. From Table $I$ it is clear that atomic resolution in a $10 \mathrm{kHz}$ bandwidth is achievable for all the detection schemes using optical beam deflection described here.

Furthermore, in practical situations interferometry and optical beam deflection have about the same sensitivity, and the measured values are a factor 10 larger than the theoretical limits set by Eqs. (2) and (6) [see $(\Delta z)_{\min }$ of setup $_{1}$ ]. That is because in our analysis we have assumed a detection system that is limited by shot noise only.

In practice many other sources contribute to the overall noise level. In an AFM mechanical and acoustic vibrations are dominant factors. Mechanical vibrations can be greatly reduced by using an air-damped optical bench, by attaching the AFM to the ceiling with elastic cords with a low elastic constant ${ }^{11}$ or by using a slab of granite on the inner tubes of car tires. Mechanical vibrations have a low frequency nature $(<100 \mathrm{~Hz})$. Acoustic noise is reduced by covering the AFM with an air-tight box ${ }^{11}$ or using an ultrahigh vacuum chamber. ${ }^{18}$ Secondly, electronic noise is created by line pickup and the power supplies for the electronics. The $50 \mathrm{~Hz}$ disturbance is the dominant factor, but also its higher harmonics contribute to the electronic noise. Low-noise signal amplifiers, stable high-voltage amplifiers, and grounding of the AFM are essential to reduce the level of electronic noise.

Laser instabilities, such as pointing instability, intensity variations, and frequency variations (e.g., mode hopping), also contribute to the noise. Furthermore, due to thermal excitation the cantilever shows random fluctuations around its equilibrium. A detailed analysis of these phenomena is given elsewhere. ${ }^{17}$

\section{CONCLUSIONS}

For various setups of the optical beam deflection technique we have calculated the signal-to-noise ratio. When applied to atomic force microscopy, the calculated signalto-noise ratios are sufficient to obtain atomic resolution.

The optical beam deflection technique is almost as sensitive as interferometry based detection systems. In practice the optical beam deflection setups use less optical components and are easier to align.

\section{APPENDIX A}

The deflection of the cantilever results into a shift of optical energy from one side of the split detector to the other side. The total optical power in a Gaussian laser beam is

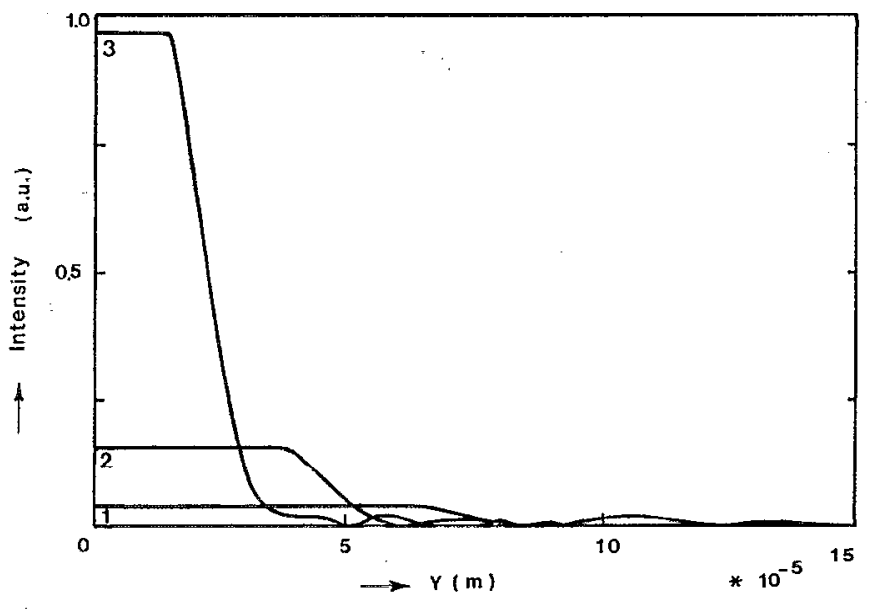

FIG. 7. Energy distributions at the detector plane in the configuration of Fig. 5 calculated using Fresnel zones. $Y$ is the radius from the center of the detector. The reflective surface at the cantilever is approximated by a circular disk with a diameter of $50 \mu \mathrm{m}$. Line 1: distance between cantilever and detector is $10 \mathrm{~mm}$, energy ratio is 0.64 ; line $2: 5 \mathrm{~mm}, 0.85$; line 3: $2 \mathrm{~mm}, 0.93$. a.u.: arbitrary units.

$$
P_{\text {tot }}=\frac{\pi}{8} D^{2} I_{0}
$$

where $I_{0}$ is the intensity at the center and $D$ is the diameter of the laser beam (intensity at $D / 2$ is $1 / e^{2}$ times $I_{0}$ ). The energy transferred

$$
\Delta P=\Delta s D I_{0}(\pi / 2)^{0.5} .
$$

Using Eq. (A1) to eliminate $I_{0}$ this results in

$$
\Delta P=4\left(\frac{2}{\pi}\right)^{1 / 2} \frac{\Delta s}{D} P_{\text {tot }}
$$

The numerical factor is set equal to $g_{2}$. Equation (A3) gives the connection between a translation of the reflected Gaussian beam at the split detector and a shift of optical energy.

\section{APPENDIX B}

In Sec. $V$ the configuration in which the laser beam is focused on the detector, is analyzed. When the distance between cantilever and detector is small, we are in the near-field region and the intensity distribution must be described by Fresnel diffraction theory. ${ }^{15}$ The problem is somewhat simplified by assuming that the phase within a Fresnel zones stays constant. In Fig. 7 graphs of the energy distributions in the detector plane resulting from these calculations are shown. The first minimum in intensity is reached at the edge of the first Fresnel zone. When the distance between cantilever and detector increases, energy is transferred from the central lobe to the side lobes and the central lobe spreads out (Fig. 7). To obtain a detection setup with a well-defined spot at the split detector, the optical power contained within the central maximum should not become smaller than $85 \%$ of the total power impinging on the detector (in the case of a Gaussian bcam, $87 \%$ of the total optical power is contained within the 
limits defined by the diameter of the beam). This ratio depends on the distance cantilever detector and on the diameter of the circular disk. As can be seen in Fig. 7 the energy distribution at the center is constant and not Gaussian. In approximation, the optical power in the central lobe $P_{\mathrm{cl}}$, is

$$
P_{\mathrm{cl}}=\frac{\pi}{4} D_{\mathrm{sd}}^{2} I_{0}
$$

$D_{s d}^{2}=4 \lambda X$ and thus is linearly dependent on $X$, the separation between cantilever and detector, and $I_{0}$ is inversely dependent on $X^{2}$. Thus $P_{\mathrm{cl}}$ is inversely dependent on $X$. The total optical power on the detector, however, stays constant. Only more energy will be contained in the side lobes when $X$ becomes larger. The change in optical power as a result of a spot displacement is described by

$$
\Delta P=2 \Delta s D_{\mathrm{sd}} I_{0}
$$

Using Eq. (B1) simple calculus shows

$$
\Delta P=\frac{8}{\pi} \frac{\Delta s}{D_{\mathrm{sd}}} P_{\mathrm{cl}}
$$

The numerical factor is denoted by $g_{3}$.

In case of Fraunhofer diffraction an Airy disk is produced at the detector. The diameter of an Airy disk is about twice the diameter of a Gaussian laser beam when originating from an aperture or beam waist having the same size. In first approximation the form of an Airy disk comes close to a Gaussian function. Because of this, the numerical factor in Eq. (A3) is substituted by $g_{2} / 2$.

\section{ACKNOWLEDGMENTS}

We thank Professor B. Bölger for a critical proofreading of this document. This work was financially supported by the Netherlands Organization for Scientific Research (NWO).

${ }^{1}$ G. Binnig, C. F. Quate, and Ch. Gerber, Phys. Rev. Lett. 56, 930 (1986).

${ }^{2}$ D. Sarid, D. Iams, V. Weissenberger, and L. S. Bell, Opt. Lett. 13, 1057 (1988).

${ }^{3}$ D. Sarid, D. Iams, J. Ingle, V. Weissenberger, and J. Ploetz, J. Vac. Sci. Technol. A 8, 378 (1990).

${ }^{4}$ Y. Martin and H. K. Wickramasinghe, Appl. Phys. Lett. 50, 1455 (1987).

${ }^{5}$ R. Erlandsson, G. M. McClelland, C. M. Mate, and S. Chiang, J. Vac. Sci. Technol. A 6, 266 (1988).

${ }_{6}^{6}$ D. Rugar, H. J. Mamin, R. Erlandsson, J. E. Stern, and B. D. Terris, Rev. Sci. Instrum. 59, 2337 (1988).

${ }^{7}$ A. J. den Boef, Appl. Phys. Lett. 55, 439 (1989).

${ }^{8}$ C. Schönenberger and S. F. Alvarado, Rev. Sci. Instrum. 60, 3131 (1989).

${ }^{9}$ D. Rugar, H. J. Mamin, and P. Guethner, Appl. Phys. Lett. 55, 2588 (1989).

${ }^{10}$ G. Meyer and N. M. Amer, Appl. Phys. Lett. 53, 2400 (1988).

${ }^{11}$ S. Alexander, L. Hellemans, O. Marti, J. Schneir, V. Elings, P. K. Hansma, M. Longmire, and J. Gurley, J. Appl. Phys. 65, 164 (1988).

${ }^{12}$ Digital Instruments, Goleta, CA.

${ }^{13}$ Park Scientific Instruments, Sunnyvale, CA.

${ }^{14}$ A. Yariv, Quantum Electronics, 3rd ed. (Wiley and Sons, New York, 1989).

${ }^{15}$ M. Born and E. Wolf, Principles of Optics (Pergamon, New York, 1964).

${ }^{16}$ C. A. J. Putman, B. G. deGrooth, Niek F. van Hulst, and J. Greve, Proceedings of STM'91 (Ultramicroscopy, in press).

${ }^{17}$ A. J. den Boef, PhD. thesis, Eindhoven, The Netherlands, 1990.

${ }^{18}$ G. Meyer and M. Amer, Appl. Phys. Lett. 56, 2100 (1990).

${ }^{19} \mathrm{G}$. Meyer and M. Amer, Appl. Phys. lett. 57, 2089 (1990).

${ }^{20}$ T. R. Albrecht, S. Akamine, T. E. Carver, and C. F. Quate, J. Vac. Sci. Technol. A 8, 3386 (1990).

${ }^{21}$ B. Drake, C. B. Prater, A. L. Weisenhorn, S. A. C. Gould, T. R. Albrecht, C. F. Quate, D. S. Cannell, H. G. Hansma, and P. K. Hansma, Science 243, 1586 (1989).

${ }^{22} \mathrm{R}$. Serber and C. H. Townes, Symposium on Quantum Electronics (Columbia University Press, New York, 1960), p. 233. 